\title{
Rigorous field theory analysis of quasiplanar waveguides
}

\author{
J. Bornemann, Dr.-Ing.
}

Indexing terms: Waveguides, Transmission lines

\begin{abstract}
An accurate field theory solution for calculating the hybrid eigenmodes of quasiplanar structures is introduced which includes both the influence of the finite strip thickness and waveguide housing grooves. Using the transverse resonance method the size of the characteristic matrix equation is kept constant even for an increasing number of discontinuities. Dispersion characteristics of various structures consisting of at least one isolated strip are presented. The fundamental $\mathrm{EH}_{0}$-modes mainly depend on the finite strip thickness, while increasing waveguide grooves lead to a significant reduction of higher-order-mode cutoff frequencies.
\end{abstract}

\section{Introduction}

Metallised substrates shielded by a rectangular waveguide have successfully been applied to millimetre-wave integrated circuits [1-4]. As numerous publications have been published dealing with dispersion characteristics of unilateral, bilateral and antipodal fin-lines, this paper is restricted to quasiplanar transmission-line configurations consisting of at least one isolated strip. Some of those cross-sections are shown in Fig. 1.

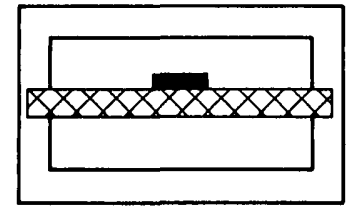

a

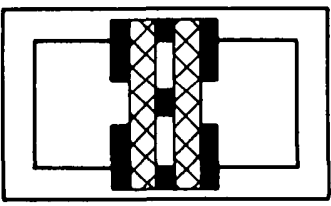

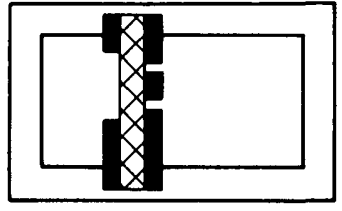

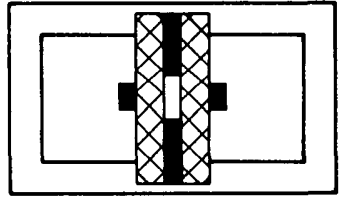

$d$
Fig. 1 Cross-sections of some quasiplanar waveguides

a Suspended substrate-line

b Shielded coplanar-slot-line

$c$ Bilateral fin-line with isolated strip-line

$d$ Two strips coupled through a fin-line slo

The most commonly used hybrid-mode analysis is the spectral domain method which, for instance, is described by Hofmann [5], Schmidt [6] and Jansen [7]. A modified finite-difference method demonstrated for the coplanar-line is presented by Schulz, Pregla [8], whose results agree well with those of Jansen [7].

Both theories neglect the influence of finite metallisation thickness and waveguide grooves to fix the substrate. The strip thickness plays a significant role in the design of microwave integrated circuits, as has been demonstrated for integrated millimetre-wave E-plane filters in References 9 and 10. Higher-order-mode cutoff frequencies are essentially reduced with increasing groove depths.

Finite strip thickness is approximately involved in Saad's and Schünemann's theory [11], but the approximation formula used becomes invalid for small slot widths together with a necessarily increasing number of eigenfunctions to be considered. The aim of this paper, therefore, is to describe an accurate hybrid-mode analysis which involves the influence of finite strip thickness and wave-

Paper 3619H (E12), received 19th August 1984

The author is with the Microwave Department, University of Bremen, Kufsteiner Strasse NWI, D-2800 Bremen 33, West Germany guide housing grooves on the dispersion characteristics. The theory utilises the transverse resonance method described in References 12, 13 and 14. The size of the characteristic matrix equation therefore does not depend on the number of discontinuities, as in classical wave propagation formulations, but is kept constant for a desired number of considered eigenfunctions. Thus, a relatively fast computer program is obtained, which calculates the normalised propagation constants for a given array of frequency sample points.

Using a superposition of electrical and magnetical vector potential functions the coupling between TE- and TM-waves is automatically involved, and so a hybrid mode generally consists of all six field components. Only in cases of homogeneous propagation media or at cutoff frequencies is the entire equation system divided into two independent systems for pure TE- and TM-modes, respectively.

\section{Theory}

Owing to the transverse resonance model used, the fundamental cross-section shown in Fig. 2 is regarded as a paral-

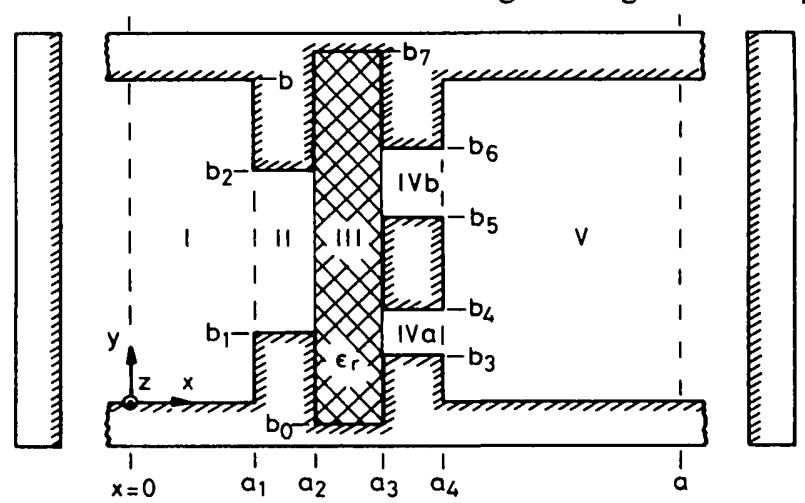

Fig. 2 Separation of hasic cross-section into homogeneous subregions lel plate line spaced by the metallised substrate. Assuming travelling waves in the $\pm x$-direction the formulation of the eigenvalue problem of the shielded planar structure is reduced to a field matching problem at the common interfaces $a_{1}$ to $a_{4}$, respectively.

Therefore, the finite strip thickness as well as the waveguide grooves can be considered in the calculations.

By subdividing the inhomogeneous cross-section according to Fig. 2 the electrumagnetic fields in each homogeneous region $i=\mathrm{I}$, II, III, IV $a, \mathrm{IV} b, \mathrm{~V}$

$$
\begin{aligned}
E^{i} & =\nabla \times A_{h z}^{i}+\frac{1}{j \omega \varepsilon^{i}} \nabla \times \nabla \times A_{e z}^{i} \\
H^{i} & =\nabla \times A_{e z}^{i}-\frac{1}{j \omega \mu} \nabla \times \nabla \times A_{h z}^{i}
\end{aligned}
$$


are derived from the $z$-components of vector potentials $A_{h}^{i}$, $\boldsymbol{A}_{e}^{i}$ which can be separated into pure cross-section functions $T_{h, e}^{i}(x, y)$ and the common propagation term $e^{-j k_{2} x_{z}}$

$$
\boldsymbol{A}_{h, e z}^{i}=T_{h, \mathrm{e}}^{i}(x, y) e^{-j k_{z} z} e_{z}
$$

with

$$
\begin{aligned}
T_{h}^{i} & =\sum_{n=0}^{\infty} Q_{h n}^{i}(x) c_{n}^{i}(y) \\
T_{e}^{i} & =\sum_{n=0}^{\infty} P_{e n}^{i}(x) s_{n}^{i}(y)
\end{aligned}
$$

The functions $c_{n}^{i}(y)$ and $s_{n}^{i}(y)$ are determined by the boundary conditions in $y$-direction yielding

$$
\begin{aligned}
c_{n}^{i}(y) & =\frac{\cos \left\{f_{n}^{i}(y)\right\}}{\sqrt{1+\delta_{o n}}} \\
s_{n}^{i}(y) & =\sin \left\{f_{n}^{i}(y)\right\} \quad \delta_{o n}=\text { Kronecker delta }
\end{aligned}
$$

with

$$
\left[\begin{array}{l}
f_{n}^{\mathrm{I}}(y) \\
f_{n}^{\mathrm{II}}(y) \\
f_{n}^{\mathrm{III}}(y) \\
f_{n}^{\mathrm{IV} a}(y) \\
f_{n}^{\mathrm{IV} b}(y) \\
f_{n}^{\mathrm{V}}(y)
\end{array}\right]=\left[\begin{array}{l}
k_{y n}^{\mathrm{I}} y \\
k_{y n}^{\mathrm{II}}\left(y-b_{1}\right) \\
k_{y n}^{\mathrm{III}}\left(y-b_{0}\right) \\
k_{y n}^{\mathrm{IV} a}\left(y-b_{3}\right) \\
k_{y n}^{\mathrm{IV} b}\left(y-b_{5}\right) \\
k_{y n}^{\mathrm{V}} y
\end{array}\right]=\left[\begin{array}{l}
\frac{n \pi}{b} y \\
\frac{n \pi}{b_{2}-b_{1}}\left(y-b_{1}\right) \\
\frac{n \pi}{b_{7}-b_{0}}\left(y-b_{0}\right) \\
\frac{n \pi}{b_{4}-b_{3}}\left(y-b_{3}\right) \\
\frac{n \pi}{b_{6}-b_{5}}\left(y-b_{5}\right) \\
\frac{n \pi}{b} y
\end{array}\right]
$$

The assumed wave propagation in the $\pm x$-direction leads to the following expressions for the functions $Q_{h n}^{i}, P_{e n}^{i}$ and their derivations:

$$
\begin{aligned}
& Q_{h n}^{i}(x)=A_{n}^{i} e^{+j k_{x n}{ }^{i} x}+B_{n}^{i} e^{-j k_{x n} i x} \\
& P_{h n}^{i}(x)=\frac{d}{d x} Q_{h n}^{i}(x)=j k_{x n}^{i}\left(A_{n}^{i} e^{+j k_{x n} i x}-B_{n}^{i} e^{-j k_{x n} i x}\right) \\
& P_{e n}^{i}(x)=\frac{1}{j k_{x n}^{i}}\left(C_{n}^{i} e^{+j k_{x n} i x}-D_{n}^{i} e^{-j k_{x n} i^{x}}\right) \\
& Q_{e n}^{i}(x)=\frac{d}{d x} P_{e n}^{i}(x)=C_{n}^{i} e^{+j k_{x n} i x}+D_{n}^{i} e^{-j k_{x n} i x}
\end{aligned}
$$

The still unknown coefficients $A_{n}^{i}, B_{n}^{i}, C_{n}^{i}, D_{n}^{i}$ are eliminated by inserting the upper $\left(x=x_{u}^{i}\right)$ and the lower $\left(x=x^{i}\right)$ boundary values in eqns. 6 and 7 . Thus the generalised transmission matrix $T^{i}$ for each subregion is obtained.

$$
\begin{aligned}
& {\left[\begin{array}{l}
\underline{P}_{h}^{i} \\
\underline{P}_{e}^{i} \\
\underline{Q}_{h}^{i} \\
\underline{Q}_{e}^{i}
\end{array}\right]=\left[\begin{array}{llll}
\underline{T}_{c}^{i} & \underline{0} & \underline{T}_{c^{\prime}}^{i} & \underline{0} \\
\underline{0} & \underline{T}_{c}^{i} & \underline{0} & \underline{T}_{s}^{i} \\
\underline{T}_{s}^{i} & \underline{0} & \underline{T}_{c}^{i} & \underline{0} \\
\underline{0} & \underline{T}_{c^{\prime}}^{i} & \underline{0} & \underline{T}_{c}^{i}
\end{array}\right] \times\left[\begin{array}{l}
P_{h}^{i} \\
\underline{P}_{e}^{i} \\
\underline{Q}_{h}^{i} \\
\underline{Q}_{e}^{i}
\end{array}\right]} \\
& x=x_{l}^{i} \\
& x=x_{u}^{i}
\end{aligned}
$$

with the diagonal matrices

$$
\begin{aligned}
& \underline{T}_{c}^{i}=\operatorname{Diag}\left\{\cos \left(k_{x n}^{i} d^{i}\right)\right\} \\
& \underline{T}_{c^{i}}^{i}=\operatorname{Diag}\left\{-k_{x n}^{i} \sin \left(k_{x n}^{i} d^{i}\right)\right\} \\
& \underline{T}_{s}^{i}=\operatorname{Diag}\left\{\frac{1}{k_{x n}^{i}} \sin \left(k_{x n}^{i} d^{i}\right)\right\} \quad d^{i}=x_{l}^{i}-x_{u}^{i}
\end{aligned}
$$

and the propagation constants $k_{x n}^{i}$ for which the relationship

$$
\begin{aligned}
\left(k_{x n}^{i}\right)^{2} & =k_{0}^{2} \varepsilon_{r}^{i}-\left(k_{y n}^{i}\right)^{2}-\left(k_{z}\right)^{2} \\
k_{0}^{2} & =\omega^{2} \mu_{0} \varepsilon_{0}
\end{aligned}
$$

holds.

It should be noticed that the wave amplitudes $P_{h}, P_{e}$, $Q_{h}$ and $Q_{e}$ in eqn. 8 are directly proportional to the field components $E_{y}, E_{z}, H_{z}$ and $H_{y}$, respectively. Matching the tangential field components of regions $i, i+1$ at the common interface $a_{i}$

$$
E_{y, z}^{i}=E_{y, z}^{i+1}, H_{y, z}^{i}=H_{y, z}^{i+1}\left(x, y \in\left\{a_{i}\right\}\right)
$$

and applying eqns. 1 and 2, eqn. 11 reads as follows:

$$
\begin{aligned}
\frac{\partial}{\partial x} T_{h}^{i} & =\frac{\partial}{\partial x} T_{h}^{i+1}+\frac{k_{z}}{\omega \varepsilon_{0}} \frac{\varepsilon_{r}^{i} / \varepsilon_{r}^{i+1}-1}{\varepsilon_{r}^{i}-\left(k_{z} / k_{0}\right)^{2}} \frac{\partial}{\partial y} T_{e}^{i+1} \\
T_{e}^{i} & =\frac{\varepsilon_{r}^{i}}{\varepsilon_{r}^{i+1}} \frac{\varepsilon_{r}^{i+1}-\left(k_{z} / k_{0}\right)^{2}}{\varepsilon_{r}^{i}-\left(k_{z} / k_{0}\right)^{2}} T_{e}^{i+1} \\
T_{h}^{i} & =\frac{\varepsilon_{r}^{i+1}-\left(k_{z} / k_{0}\right)^{2}}{\varepsilon_{r}^{i}-\left(k_{z} / k_{0}\right)^{2}} T_{h}^{i+1} \\
\frac{\partial}{\partial x} T_{e}^{i} & =\frac{\partial}{\partial x} T_{e}^{i+1}+\frac{k_{z}}{\omega \mu_{0}} \frac{\varepsilon_{r}^{i}-\varepsilon_{r}^{i+1}}{\varepsilon_{r}^{i}-\left(k_{z} / k_{0}\right)^{2}} \frac{\partial}{\partial y} T_{h}^{i+1}
\end{aligned}
$$

The second terms in eqns. 12 and 15 denote the coupling between TE- and TM-modes. It is remarkable that the coupling vanishes in cases of homogeneous propagation media $\left(\varepsilon_{r}^{i+1}=\varepsilon_{r}^{i}\right)$ and at the cutoff frequencies of the hybrid modes $\left(k_{z}=0\right)$.

As $c_{n}^{i}(y)$ and $s_{n}^{i}(y)$ constitute systems of orthogonal functions, eqns. $12-15$ can be expressed in terms of the wave amplitude vectors according to eqn. 8 . This procedure provides coupling matrices $\underline{K}^{i, i+1}$ which are explained in the Appendix.

Finally the multiplicative combination of transmission and coupling matrices

$$
\begin{aligned}
& {\left[\begin{array}{l}
\underline{P}_{h}^{l} \\
\underline{P}_{e}^{I} \\
\underline{Q}_{h}^{l} \\
\underline{Q}_{e}^{!}
\end{array}\right]=\underline{T}^{I} \prod_{i=11}^{\mathrm{V}} K^{i-1, i} \underline{T}^{i}} \\
& x=0
\end{aligned}
$$

and the still missing boundary conditions at the metallic surfaces $\left(P_{h, e}^{\mathrm{I}, \mathrm{v}}=\underline{0}\right.$ at $\left.x=0, a\right)$, lead to the characteristic matrix which is the upper right quarter of the matrix product in eqn. 16 and which has to be solved numerically for the zeros of its determinant. For the computer calculations consideration of the first eighteen terms of the infinite series in eqn. 3 is sufficient.

\section{Results}

Fig. $3 a$ shows the dispersion characteristics of the first eight propagating eigenmodes on a suspended substrate configuration. Considering a low strip thickness of $t=$ $5 \mu \mathrm{m}$ the values calculated are in agreement with those presented by Hofmann [5] for $t=0$ and the fundamental $\left(\mathrm{EH}_{0}\right)$ mode. Increasing the strip thickness, however, leads to decreasing propagation constants (Fig. $3 b$ ), because the electromagnetic field is mainly concentrated between the strip and the upper waveguide wall as the substrate is kept at middle waveguide height. The effects of this parameter 
on higher-order-mode cutoff frequencies are within $-4.9 \%$ and $+4.4 \%$ for HE- and EH-modes, respectively.

The influence of the waveguide grooves is demonstrated
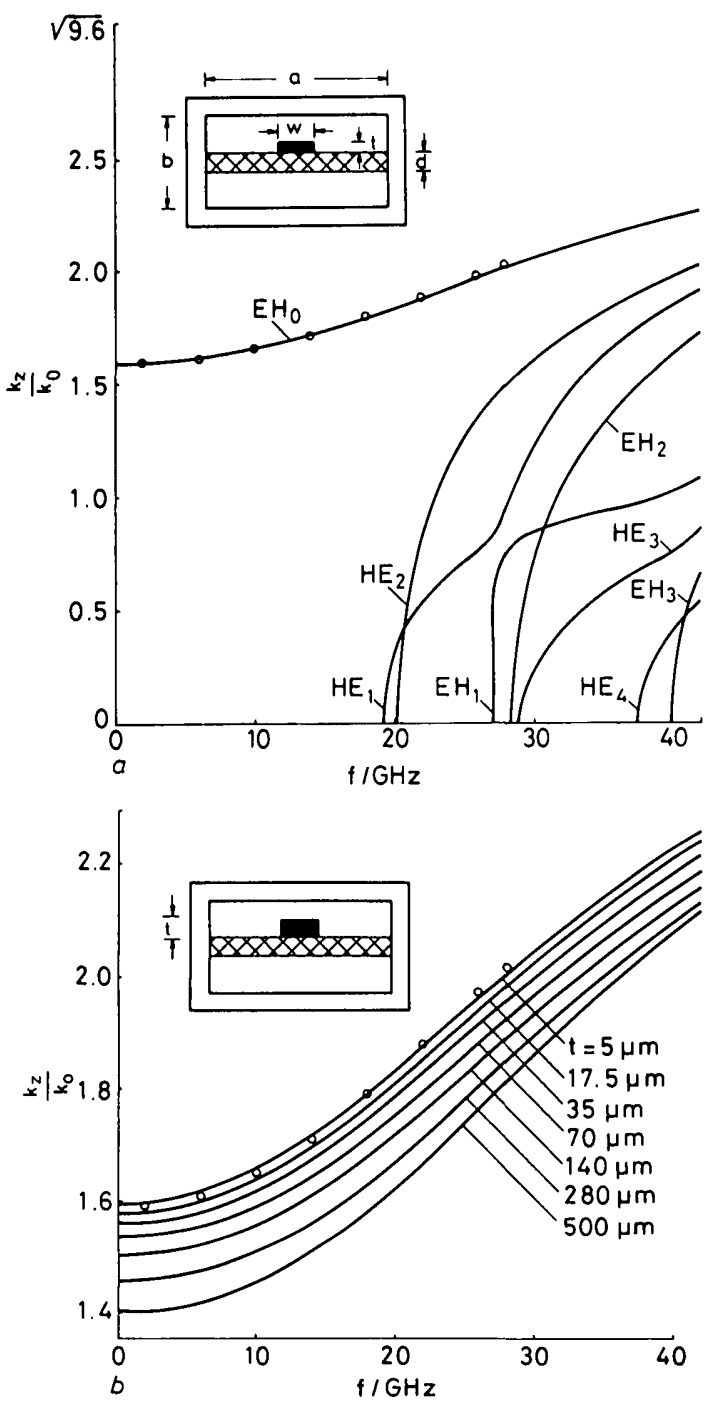

Fig. 3 Suspended substrate dispersion behaviour calculated by authors method and by Hofmann's method [5]

$a$ Dispersion characteristics

$a=2 b=7.112 \mathrm{~mm}$

$d=635 \mu \mathrm{m}, t=5 \mu \mathrm{m}$

$w=1 \mathrm{~mm}, \varepsilon_{r}=9.6$

$b$ Influence of strip thickness on fundamental $\mathrm{EH}_{0}$-mode propagation constan

- Authors method $(t=5 \mu \mathrm{m})$

O०O Hoffmans method $[5](t=0)$

in Figs. 4. As long as the groove depth is neglected the calculated dispersion characteristics (dashed lines in Fig. $4 a$ ) agree well with investigations by Schmidt [6]. Increasing the groove depths, however, leads to decreasing higherorder-mode cutoff-frequencies, especially of the $\mathrm{HE}_{2}-$ and $\mathrm{HE}_{3}$-modes, as shown in Fig. $4 a$ (solid lines) and Fig. $4 b$. If a $T E_{10}$-wave of an equivalent empty waveguide (WR 12) is incident, the first hybrid modes to be excited are $\mathrm{HE}_{1}$ and $\mathrm{HE}_{3}$, for reasons of symmetry $[3,6]$, whereas the $\mathrm{EH}_{0}$-and $\mathrm{HE}_{2}$-modes are excited by a $\mathrm{TE}_{01}$-wave. Therefore, the applicable bandwidths of modes $\mathrm{EH}_{0}$ and $\mathrm{HE}_{1}$ are significantly reduced by the $\mathrm{HE}_{2}$ - and $\mathrm{HE}_{3}$-mode cutoff frequencies, respectively, which are influenced by the groove depth. The influence of this real structure parameter on the $\mathrm{EH}_{0^{-}}$and $\mathrm{HE}_{1}$-propagation constants is inessential (Fig. $4 a$ ), which is in accordance with fin-line results presented in Reference 14 and which is in contrast to investigations by Beyer [15], who calculated a deviation of up to $-5 \%$ related to the effective dielectric constant of the fundamental $\mathrm{HE}_{1}$-mode of an unilateral fin-line. For the small slot widths considered in Figs. 4 the electromagnetic fields of both modes are mainly concentrated within the slot areas. Therefore, it is evident that their propagation constants cannot depend essentially on the groove depth.
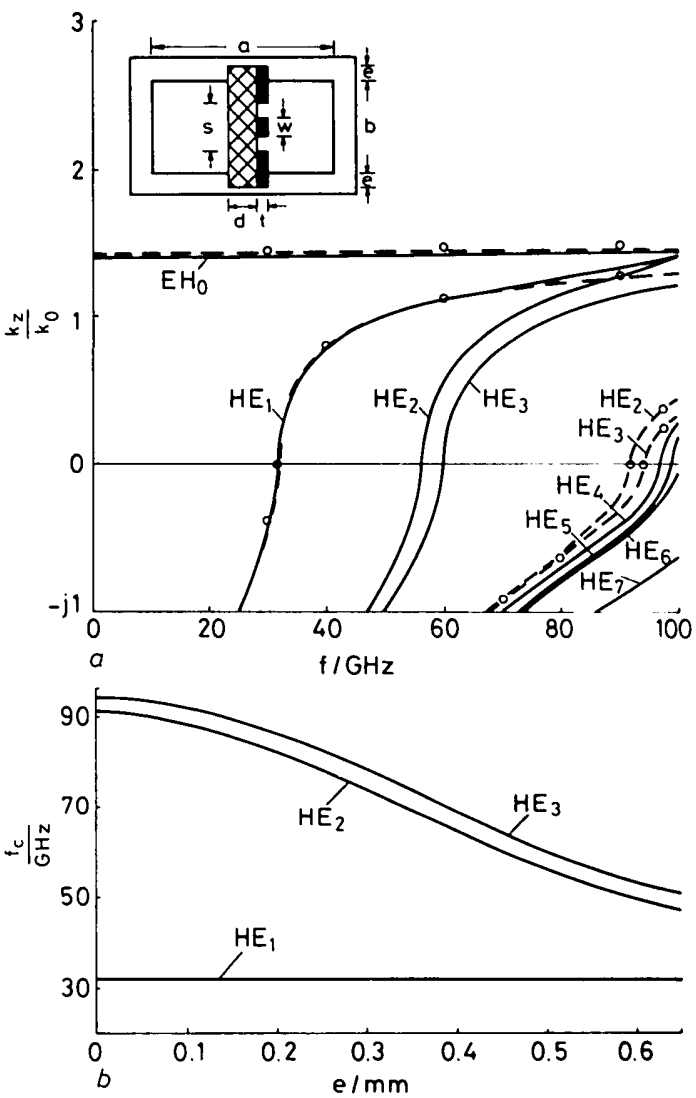

Fig. 4 Coplanar waveguide dispersion characteristics calculated by this method and by Schmidt's method [6]

$a$ Dispersion characteristics

- D - $e=0$, this method

$e=0.5 \mathrm{~mm}$, this method

OOO $e=t=0$, Schmidt's method (graphical reproduction from Reference 6) $a=2 b=3.1 \mathrm{~mm}$

$d=220 \mu \mathrm{m}, t=5 \mu \mathrm{m}$

$w=0.2 \mathrm{~mm}, s=0.6 \mathrm{~mm}$

$\varepsilon_{r}=3.75$

$r$
$b$ Influence of waveguide groove depth on higher-order-mode cutoff frequencies

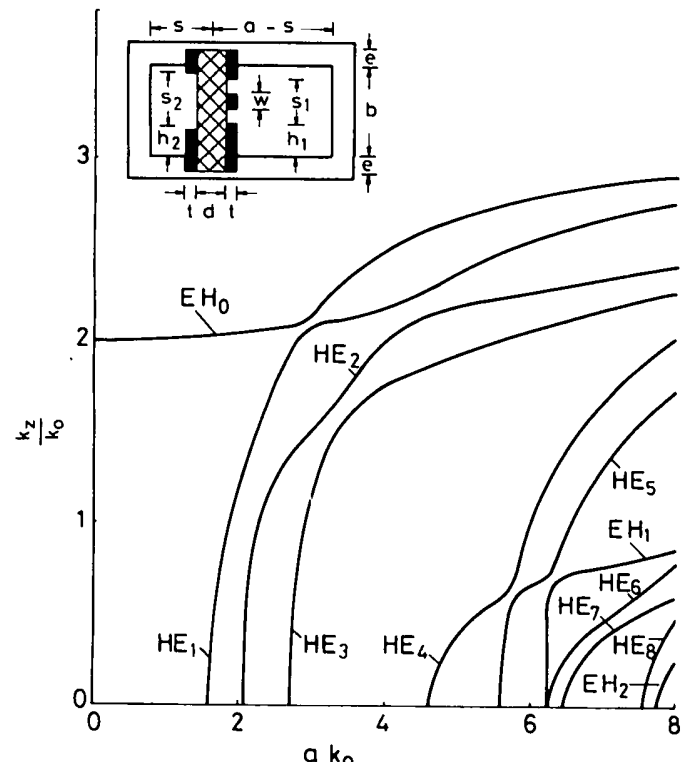

Fig. 5 Normalised propagation constants $k_{z} / k_{0}$ of the first eleven propagating hybrid eigenmodes on an unsymmetrical shielded coplanar-slot-line against normalised frequency $a k_{0}$

$b / a=0.5, d / a=e / a=0.1$

$t / a=0.01, w / a=\frac{1}{12}$

$s / a=0.25, \varepsilon_{r}=10$

$h_{1} / a=1 / 6, s_{1} / a=\frac{3}{12}$

$h_{2} / a=\frac{1}{8}, s_{2} / a=\frac{5}{16}$ 
The efficiency of the transverse resonance method is demonstrated in Fig. 5 for an unsymmetrical cross-section. There appears to be a mutual influence of the hybrid eigenmodes resulting in nontypical dispersion curves, whereas this behaviour is not observed in symmetrical structures as shown in Fig. 6.

Fig. 7 shows the dispersion characteristics of two strips

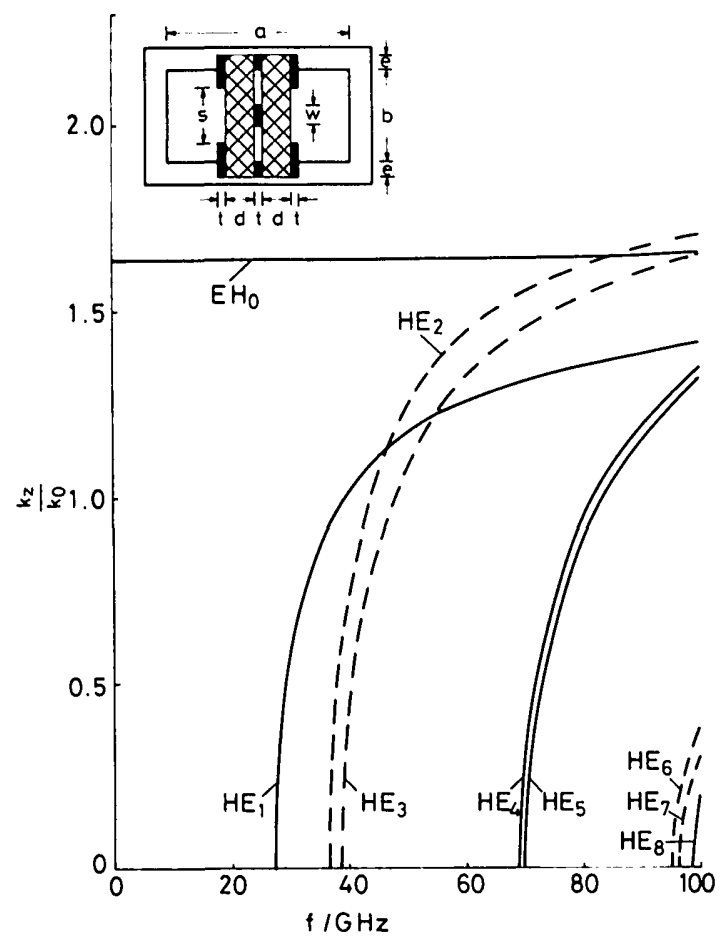

Fig. 6 Dispersion characteristics of the first nine propagating hybrid eigenmodes on a bilateral fin-line with isolated strip-line

magnetic wall, $\quad$ electric wall at $a / 2$

$a=2 b=3.1 \mathrm{~mm}$

$d=220 \mu \mathrm{m}, t=5 \mu \mathrm{m}$

$w=0.2 \mathrm{~mm}, s=0.6 \mathrm{~mm}$

$e=0.5 \mathrm{~mm}, \varepsilon_{\mathrm{r}}=3.75$

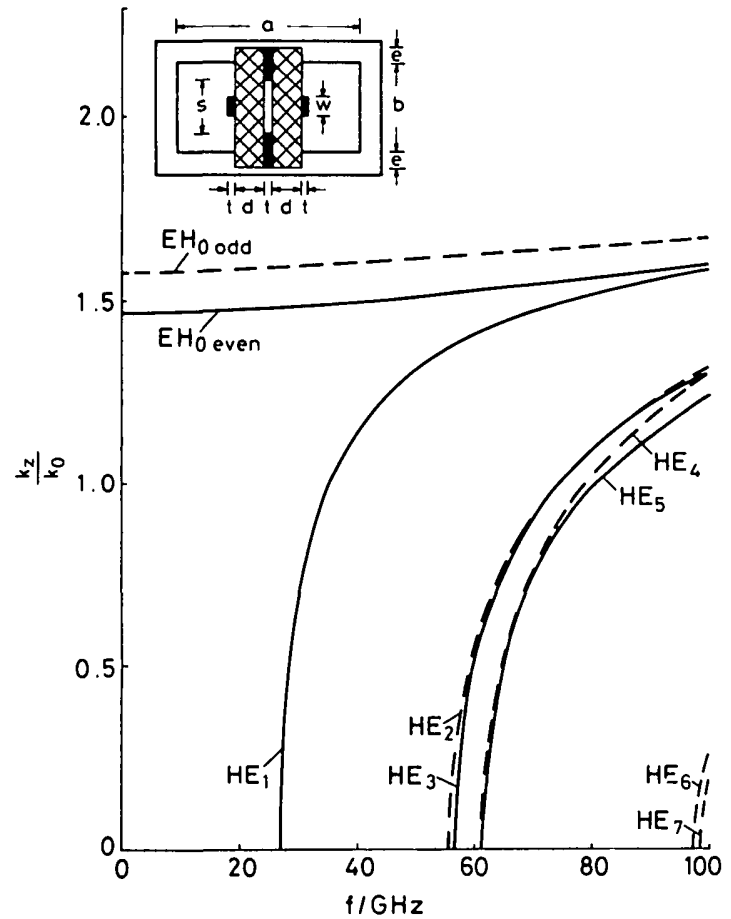

Fig. 7 Dispersion characteristics of two strips coupled through the slot of a fin-line

- magnetic wall, ....- electric wall at $a / 2$

$a=2 b=3.1 \mathrm{~mm}$

$d=220 \mu \mathrm{m}, t=5 \mu \mathrm{m}$

$w=0.2 \mathrm{~mm}, s=0.6 \mathrm{~mm}$

$e=0.5 \mathrm{~mm}, \varepsilon_{r}=3.75$ coupled through the slot of a fin-line. These structures are applicable to integrated directional couplers below $\mathrm{HE}_{1}$-cutoff. They may be used as codirectional couplers if a significant difference in the even and odd fundamentalmode phase velocities occurs, which is achieved by increasing the slot width (Fig. 8).

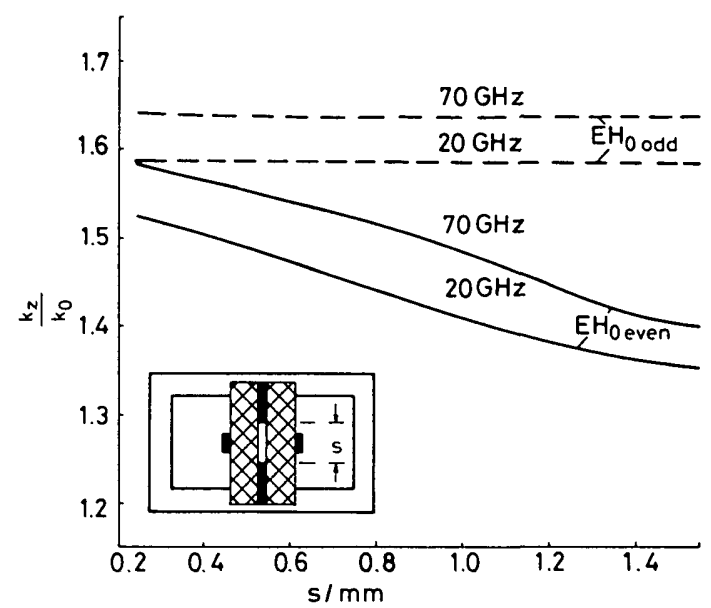

Fig. 8 Even and odd fundamental mode normalised propagation constants against slot width $s$

$a=2 b=3.1 \mathrm{~mm}$

$d=220 \mu \mathrm{m}, t=5 \mu \mathrm{m}$

$w=0.2 \mathrm{~mm}$

$e=0.5 \mathrm{~mm}, \varepsilon_{r}=3.75$

Contradirectional coupling is only possible at small deviations of the related phase velocities so that the structure can be regarded as nearly homogeneous. Indeed, this is obtained by horizontally separating the strips (Fig. 9),

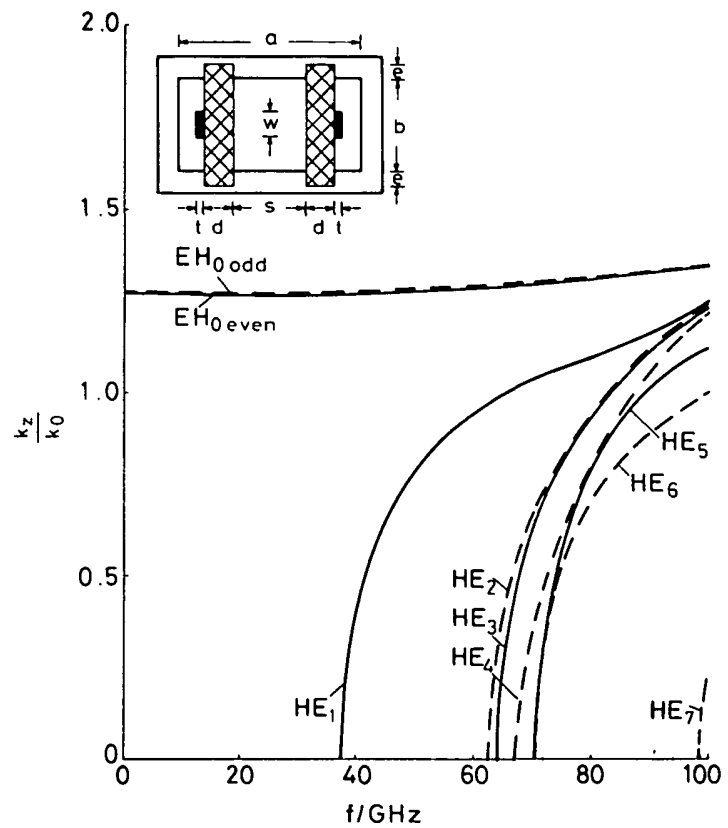

Fig. 9 Dispersion characteristics of two E-plane suspended substratelines

- magnetic wall, $--\cdots$ - electric wall at $a / 2$

$a=2 b=3.1 \mathrm{~mm}$

$d=220 \mu \mathrm{m}, t=5 \mu \mathrm{m}$

$w=0.5 \mathrm{~mm}, s=a / 2-d$

$e=0.5 \mathrm{~mm}, \varepsilon_{r}=3.75$

but the coupling is then also reduced. Pure contradirectional couplers in shielded broadside coupled-strip technology, therefore, are applicable if extremely loose coupling is desired.

\section{Conclusion}

A rigorous hybrid-mode dispersion analysis is described for quasiplanar waveguides. The transverse resonance

IEE PROCEEDINGS, Vol. 132, Pt. H, No. I, FEBRUARY 1985 
method used exhibits the advantage of keeping the size of the characteristic matrix equation constant, even for an increasing number of discontinuities. Real structure parameters, such as finite metallisation thickness and groove depths needed to fix the substrate, may be investigated by this method as well as symmetrical structures which follow from the basic configuration of a shielded coplanar-slotline.

Dispersion characteristics of various structures consisting of at least one isolated strip are presented. The propagation constants of the fundamental $\mathrm{EH}_{0}$-modes are decreased by the finite strip thickness, while increasing waveguide grooves lead to a limitation of monomode bandwidths since higher-order-mode cutoff frequencies are significantly reduced.

To avoid atypical dispersion behaviour (e.g. see Fig. 5) symmetrical configurations should be preferred for practical application, if possible. Broadside coupled strips in a rectangular waveguide housing are applicable in integrated microwave directional couplers below $\mathrm{HE}_{1}$-cutoff. Codirectional (quasi-TEM-wave) couplers should, however, be used if extremely loose coupling is desired.

\section{References}

1 MEIER, P.J.: 'Integrated fin-line millimeter components', IEEE Trans., 1974, MTT-22, pp. 1209-1216

2 MEIER, P.J.: 'Millimeter integrated circuits suspended in the E-plane of rectangular waveguide', ibid., 1978, MTT-26, pp. 726-733

3 ADELSECK, B., CALLSEN, H., HOFMANN, H., MEINEL, H., and REMBOLD, B.: 'Neue Millimeterwellenkomponenten in quasiplanarer Leitungstechnik', Frequenz 35, 1981, 5, pp. 118-123

4 ARNDT, F., BORNEMANN, J., GRAUERHOLZ, D., and VAHLDIECK, R.: 'Theory and design of low-insertion-loss fin-line filters', IEEE Trans., 1982, MTT-30, pp. 155-163
5 HOFMANN, H.: 'Dispersion of planar waveguides for millimeterwave application', $A E \ddot{U} B d .3 I, 1977,1$, pp. $40-44$

6 SCHMIDT, L.-P.: 'A comprehensive analysis of quasiplanar waveguides for millimeter-wave application'. Proc. 11 th European microwave conf., Amsterdam, 1981, pp. 315-320

7 JANSEN, R.H.: 'Unified user-oriented computation of shielded, covered and open planar microwave and millimeterwave transmission-line characteristics, IEE J. Microwates, Optics and Acoustics, 1979, 3, (1), pp. 14-22

8 SCHULZ, U.L., and PREGLA, R.: 'A new technique for the analysis of the dispersion characteristics demonstrated for the coplanar line'. Proc. 10th European Microwave Conf., Warsaw, 1980, pp. 331-335

9 BORNEMANN, J., VAHLDIECK, R., ARNDT, F., and GRAUERHOLZ, D.: 'Optimized low-insertion-loss millimeter-wave fin-line and metal insert filters', Radio \& Electron. Eng., 1982, 51, pp. 513-521

10 VAHLDIECK, R., BORNEMANN, J., ARNDT, F., and GRAUERHOLZ, D.: 'Optimized waveguide E-plane metal insert filters for millimeter-wave applications', IEEE Trans., 1983, MTT-31, pp. 65-69

11 SAAD, A.M.K., and SCHONEMANN, K.: 'Efficient eigenmode analysis for planar transmission lines', IEEE Trans., 1982, MTT-30, pp. $2125-2131$

12 FRITZSCHE, H.: 'Die frequenzabhängigen Úbertragungseigenschaften gekoppelter Streifenleitungen im geschichteten Dielektrikum', 1973, NTZ 26, (1), pp. 1-8

13 ARNDT, F., and PAUL, G.-U.: 'The reflection definition of the characteristic impedance of microstrips', IEEE Trans., 1979, MTT-27, pp. 724-731

14 VAHLDIECK, R.: 'Accurate hybrid mode analysis of various fin-line configurations including multilayered dielectrics, finite metallization thickness and substrate holding grooves', IEEE Trans., 1984. MTT-32, pp. 1454-1460

15 BEYER, A.: 'Analysis of the characteristics of an earthed fin line', IEEE Trans., 1980, MTT-29, pp. 676-680

\section{Appendix}

The coupling matrices $\underline{K}^{\text {1, "I }}$ and $\underline{K}^{\text {"1, III }}$ in eqn. 16 read as follows

$$
\begin{aligned}
& \underline{K}^{1, ~ I I}=\left[\begin{array}{cccc}
\frac{2}{b} \underline{J}_{c}^{1 . \prime \prime} & \underline{0} & \underline{0} & \underline{0} \\
\underline{0} & \frac{2}{b} \underline{J}_{s}^{1, \text { II }} & \underline{0} & \underline{0} \\
\underline{0} & \underline{0} & \frac{b_{2}-b_{1}}{2}\left(\underline{J}_{c}^{1, I^{T}}\right)^{-1} & \underline{0} \\
\underline{0} & \underline{0} & \underline{0} & \frac{b_{2}-b_{1}}{2}\left(\underline{J}_{s}^{1, I^{T}}\right)^{-1}
\end{array}\right]
\end{aligned}
$$

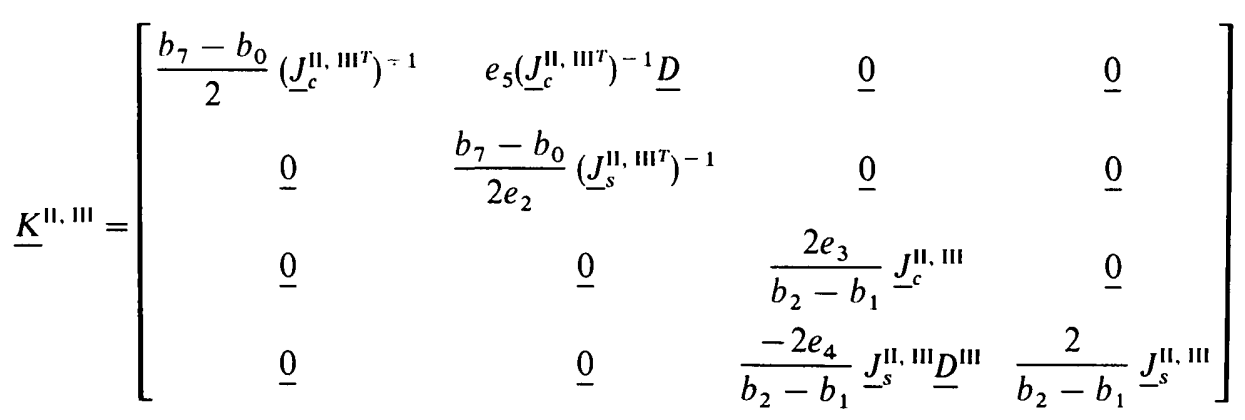

Since the entire number of modes in subregions IV equals the number of modes in regions III and $V$ $\left(N^{\mathrm{lV} a}+N^{\mathrm{IV} b}=N^{\mathrm{lll}}=N^{\mathrm{V}}\right) \underline{K}^{\mathrm{III}, \mathrm{IV}}$ and $\underline{K}^{\mathrm{IV}, \mathrm{v}}$ are determined by submatrices

$$
\underline{K}^{\mathrm{III}, \mathrm{IV}}=\left[\begin{array}{cc}
\underline{E}^{\mathrm{III}, \mathrm{IV}} & \underline{0} \\
\underline{0} & \left(\underline{H}^{\mathrm{III}, \mathrm{IV}}\right)^{-1}
\end{array}\right] ; \quad \underline{K}^{\mathrm{IV}, \mathrm{v}}=\left[\begin{array}{cc}
\left(\underline{E}^{\mathrm{IV}, \mathrm{v}}\right)^{-1} & \underline{0} \\
\underline{0} & \underline{H}^{\mathrm{v}, \mathrm{v}}
\end{array}\right]
$$

where

$$
\underline{E}^{\mathrm{III}, \mathrm{IV}}=\frac{2}{b_{7}-b_{0}}\left[\begin{array}{ccll}
\underline{J}_{c}^{\mathrm{III}, \mathrm{IV} a} & \underline{J}_{c}^{\mathrm{III}, \mathrm{IV} b} & e_{1} \underline{J}_{c}^{\mathrm{III}, \mathrm{IV} a} \underline{D}^{\mathrm{IV} a} & e_{1} \underline{J}_{c}^{\mathrm{III}, \mathrm{IV} b} \underline{D}^{\mathrm{IV} b} \\
\underline{0} & \underline{0} & e_{2} \underline{J}_{s}^{\mathrm{III}, \mathrm{IV} a} & e_{2} \underline{J}_{s}^{\mathrm{IIN}, \mathrm{VV} b}
\end{array}\right]
$$


$\underline{H}^{\mathrm{III}, \mathrm{IV}}=\left[\begin{array}{lc}\frac{2 e_{3}}{b_{4}-b_{3}}\left(\underline{J}_{c}^{\mathrm{III}, \mathrm{IV} a}\right)^{T} & \underline{0} \\ \frac{2 e_{3}}{b_{6}-b_{5}}\left(\underline{J}_{c}^{\mathrm{III}, \mathrm{IV} b}\right)^{T} & \underline{0} \\ \frac{-2 e_{4}}{b_{4}-b_{3}}\left(\underline{J}_{s}^{\mathrm{III}, \mathrm{IV} a}\right)^{T} \underline{D}^{\mathrm{III}} & \frac{2}{b_{4}-b_{3}}\left(\underline{J}_{s}^{\mathrm{IIII}, \mathrm{IV} a}\right)^{T} \\ \frac{-2 e_{4}}{b_{6}-b_{5}}\left(\underline{J}_{s}^{\mathrm{IIII}, \mathrm{IV} b}\right)^{T} \underline{D}^{\mathrm{IIII}} & \frac{2}{b_{6}-b_{5}}\left(\underline{J}_{s}^{\mathrm{III}, \mathrm{IV} b}\right)^{T}\end{array}\right]$

$\underline{E}^{\mathrm{IV}, \mathrm{v}}=\frac{b}{2}\left[\begin{array}{cccc}\underline{J}_{c}^{\mathrm{IV} a, \mathrm{v}} & \underline{J}_{c}^{\mathrm{IV} b, \mathrm{v}} & \underline{0} & \underline{0} \\ \underline{0} & \underline{0} & \underline{J}_{s}^{\mathrm{IV} a, \mathrm{v}} & \underline{J}_{s}^{\mathrm{IV} b, \mathrm{v}}\end{array}\right]$

$\underline{H}^{\mathrm{IV}, \mathrm{v}}=\left[\begin{array}{cc}\frac{b_{4}-b_{3}}{2}\left(\underline{J}_{c}^{\mathrm{IV} a, \mathrm{v}}\right)^{T} & \underline{0} \\ \frac{b_{6}-b_{5}}{2}\left(\underline{J}_{c}^{\mathrm{IV} b, \mathrm{v}}\right)^{T} & \underline{0} \\ \underline{0} & \frac{b_{4}-b_{3}}{2}\left(\underline{J}_{s}^{\mathrm{IV} a, \mathrm{v}}\right)^{T} \\ \underline{0} & \frac{b_{6}-b_{5}}{2}\left(\underline{J}_{s}^{\mathrm{IV} b, \mathrm{v}}\right)^{T}\end{array}\right]$

The abbreviations $e_{1}$ to $e_{5}$ are

$$
\begin{array}{ll}
e_{1}=\frac{k_{z}}{\omega \varepsilon_{0}} \frac{1}{\varepsilon_{r}-\left(k_{z} / k_{0}\right)^{2}} & e_{2}=\varepsilon_{r} \frac{1-\left(k_{z} / k_{0}\right)^{2}}{\varepsilon_{r}-\left(k_{z} / k_{0}\right)^{2}} \\
e_{3} & =\frac{\varepsilon_{r}-\left(k_{z} / k_{0}\right)^{2}}{1-\left(k_{z} / k_{0}\right)^{2}} \quad e_{4}=\frac{k_{z}}{\omega \mu_{0}} \frac{\varepsilon_{r}-1}{1-\left(k_{z} / k_{0}\right)^{2}} \\
e_{5} & =\frac{k_{z}}{\omega \varepsilon_{0}} \frac{1 / \varepsilon_{r}-1}{1-\left(k_{z} / k_{0}\right)^{2}}
\end{array}
$$

Diagonal matrices

$$
\begin{aligned}
\underline{D} & =\operatorname{Diag}\left(\frac{n \pi}{2}\right) & \underline{D}^{\mathrm{III}} & =\operatorname{Diag}\left(\frac{n \pi}{b_{7}-b_{0}}\right) \\
\underline{D}^{\mathrm{IVa} a} & =\operatorname{Diag}\left(\frac{n \pi}{b_{4}-b_{3}}\right) & \underline{D}^{\mathrm{IV} b} & =\operatorname{Diag}\left(\frac{n \pi}{b_{6}-b_{5}}\right)
\end{aligned}
$$

Coupling integrals

$$
\begin{aligned}
\left(\underline{J}_{c}^{\mathrm{I}}, \mathrm{II}\right)_{n k} & =\int_{b_{1}}^{b_{2}} c_{n}^{\mathrm{I}}(y) c_{k}^{\mathrm{II}}(y) d y \\
\left(\underline{J}^{\mathrm{II}}, \mathrm{III}\right)_{n k} & =\int_{b_{1}}^{b_{2}} c_{n}^{\mathrm{II}}(y) c_{k}^{\mathrm{III}}(y) d y \\
\left(\underline{J}^{\mathrm{III}, \mathrm{IV} a}\right)_{n k} & =\int_{b_{3}}^{b_{4}} c_{n}^{\mathrm{III}}(y) c_{k}^{\mathrm{IV} a}(y) d y \\
\left(\underline{(}_{c}^{\mathrm{III}, \mathrm{IV} b}\right)_{n k} & =\int_{b_{5}}^{b_{6}} c_{n}^{\mathrm{III}}(y) c_{k}^{\mathrm{IV} b}(y) d y \\
\left(\underline{J}_{c}^{\mathrm{IV} a, \mathrm{~V}}\right)_{n k} & =\int_{b_{3}}^{b_{4}} c_{n}^{\mathrm{V}}(y) c_{k}^{\mathrm{IV} a}(y) d y \\
\left(\underline{J}_{c}^{\mathrm{IV} b, \mathrm{~V}}\right)_{n k} & =\int_{b_{5}}^{b_{6}} c_{n}^{\mathrm{V}}(y) c_{k}^{\mathrm{IV} b}(y) d y
\end{aligned}
$$

For coupling integrals $\underline{J}_{s}$, replace $c_{n}(y)$ by $s_{n}(y)$. 